\title{
Diagnosis of cardiac structural changes: Utility of altered gadolinium kinetics on cardiac magnetic resonance
}

We read with great interest the article by Dwivedi et al. [1] entitled "Progressive breathlessness in an Afro Caribbean hypertensive subject", which was published in the previous issue of "Cardiology Journal”.

They aimed to show diagnostic utility of hyper-enhancement patterns on cardiac magnetic resonance (CMR) in differentiating a range of structural changes that manifest as increased wall thickness. They also mentioned that primary cardiac amyloidosis could not be excluded on the basis of the T1 mapping findings. This case highlights the diagnostic abilities of CMR in questionable cases, yet we have some comments about the article.

In addition to the enhancement pattern, myocardial tagging magnetic resonance images can show any active displacement and deformation of tags in regions conflicting with septal hypertrophy.

In amyloid patients, differences in $\mathrm{T} 1$ between subepicardium, myocardium, subendocardium, and blood are present. Gadolinium washout from blood and myocardium is significantly faster in amyloid patients, resulting in a higher blood $\mathrm{T} 1$ over time $[2,3]$.

In conclusion, late gadolinium enhancement provides incremental information for differentiating a range of structural changes. Subendocardial T1 over time is significantly lower than blood and myocardium ones, and diagnosis can be established using the combination of enhancement pattern and subendocardial-blood T1 difference with $>90 \%$ concordance.

Conflict of interest: none declared

\section{References}

1. Dwivedi G, MacFadyen RJ, Varma C, Sharma V, Neil D, Steeds RP. Progressive breathlessness in an Afro Caribbean hypertensive subject. Cardiol J, 2012; 19: 646-649.

2. Hombach V, Merkle N, Bernhard P, Rasche V, Rottbauer W. Prognostic significance of cardiac magnetic resonance imaging: Update 2010. Cardiol J, 2010; 17: 549-557.

3. Maceira AM, Joshi J, Prasad SK et al. Cardiovascular magnetic resonance in cardiac amyloidosis. Circulation, 2005; 111: 186-193.

Emre Yalcinkaya ${ }^{1}$, Baris Bugan ${ }^{2}$, Murat Celik, Uygar Cagdas Yuksel

${ }^{1}$ Gulhane Military Medical Faculty, Department of Cardiology,

GATA Etlik 06018 Ankara, Turkey,

tel: +903123044257 , fax: +903123044250 ,

e-mail: dremreyalcinkaya@gmail.com

${ }^{2}$ Malatya Army Hospital, Department of Cardiology, Malatya, Turkey 